\title{
Controversial data on the association of Aeromonas with diarrhoea in a recent Hong Kong study
}

In a recent issue of this journal, a study performed in Hong Kong by Chu et al. (2006) concluded that there was no association between the presentation of diarrhoeal symptoms and the faecal isolation of Aeromonas spp. among outpatients from public clinics. Using a multiplex PCR, the authors screened for the presence of three haemolysin genes (ahhl, asal and aerA) in order to genotype 253 Aeromonas strains isolated in the investigation of gastroenteritis. No significant association was found between the genotypes, species identification and the amount of growth (on the primary culture), and the presentation of diarrhoeal symptoms defined as bloody, mucous or watery stool specimens.

In our opinion, the authors derived a highly unsubstantiated conclusion from very limited data on a topic that has been under discussion and heavily researched by aeromonad specialists for many years worldwide. In our view, the most important limitations of the study were the criteria on which the authors based their conclusions, which were (1) the presence or absence of the three haemolysin genes, (2) the type of stool as a direct indication of the presence of diarrhoeal symptoms, and (3) the amount of bacteria as an indication of diarrhoea. First, the Canadian PCR genotyping approach (Wang et al., 2003) used by Chu and others was not designed to determine the role that specific strains may play in the development of diarrhoea, but rather was set up simply to screen strains for specific haemolysin genes. The presence or absence of any particular haemolytic virulence gene(s) is only partial evidence of the virulence potential of that strain, and non-haemolytic strains can also be implicated in human infection (Wang et al., 2003). In addition, the authors' results may not even reflect the true presence or absence of the genes studied because, as stated by Wang et al. (2003), PCR may have a low sensitivity. Negative
PCR results therefore have to be validated by DNA hybridization with the specific probes. It is well known that the virulence of Aeromonas, as with many other pathogenic micro-organisms, is multifactorial and very complex. Strains may produce different toxins with haemolytic, cytotoxic and enterotoxic activity, as well as many enzymes that are considered virulence factors, and may even possess a type III secretion system (Chopra \& Houston, 1999; Chacón et al., 2003; Galindo et al., 2006). No mention is made in the study of Chu and others of the significant body of published work on this subject, nor of the three enterotoxins, Act, Ast and Alt, that have clearly been implicated in cases of Aeromonas-associated diarrhoea (Albert et al., 2000). The considerable array of virulence factors that have been described in Aeromonas has recently been reviewed (Galindo et al., 2006), and many of these have been identified on the recently sequenced genome of the type strain Aeromonas hydrophila ATCC 7966 (Seshadri et al., 2006). However, the specific role that each of these putative virulence factors, alone or in combination, may play in the development of diarrhoea is unknown.

In relation to the other two criteria used by Chu and others, it is currently accepted that more important than the consistency/ aspect of the faeces or the amount of Aeromonas recovered from them, both regarded as key characters by the authors, is the evolution of the diarrhoeal process and resolution of the symptoms with the clearance of the microbe from the stool. These aspects were completely neglected in their study. So far, little is known about the amount of bacteria present in the faeces and its relationship with the severity of the disease. The intestine is a complex ecosystem in which the host plays a major role in controlling levels of individual species, so levels vary greatly and depend on the host (Teunis et al., 1999). In a retrospective study of traveller's diarrhoea, we found a $2 \%$ incidence of Aeromonas, and evidence that the persistence of symptoms made antibiotic treatment necessary (Vila et al., 2003). Unfortunately, data on the need for antibiotic therapy or rehydration were missing in the study of Chu and others, but these are factors that could have provided additional insights into the severity of the diarrhoea. One of the most important shortcomings of the correspondence article of Chu and others is that information on the type of patients (adults, children or both) included in the study, and the presence or absence of underlying disease, together with a true definition of diarrhoea, were lacking. The American College of Gastroenterology has outlined potential signs of infectious diarrhoea and recommends performing stool cultures in adults in the presence of severe diarrhoea (at least six times in a $24 \mathrm{~h}$ period), a temperature $\geqslant 38.5{ }^{\circ} \mathrm{C}$, passage of bloody stools or persistent diarrhoea ( $\geqslant 3$ days). The precise application of these strict criteria were retrospectively analysed in a recent Hong Kong study of acute bacterial gastroenteritis in adults attending the emergency department of a major hospital (Chan et al., 2003). This study revealed that Aeromonas was isolated in $6.9 \%$ of cases, in $88.9 \%$ of which stool cultures were performed because the strict criteria were followed (Chan et al., 2003).

Chu and others have already highlighted some limitations of their own study, i.e. the lack of more epidemiological data from the patients, and the possibility that the aspect of the faeces had changed. In our view, these limitations are very important and make it hard to substantiate their conclusions. We think it is important for the readers of the journal to look at other interpretations and conclusions published on this subject worldwide. In this sense, the association of Aeromonas and diarrhoea symptoms in both children and adults has been described in studies from all over the world (Janda \& Abbott, 1998; Chan \& Ng, 2004; Figueras, 2005; 
Martin-Carnahan \& Joseph, 2005; Subashkumar et al., 2006). The latest studies have reported that $2-20 \%$ of the diarrhoeal cases from children around the world are induced solely by species of Aeromonas, and that only $0-2 \%$ of children that harbour Aeromonas do not present with diarrhoeal symptoms (Galindo et al., 2006). A recent Brazilian report of a large acute diarrhoeal outbreak of 2170 cases revealed that Aeromonas species were recovered in $19.5 \%$ of cases (Hofer et al., 2006).

A few, weak arguments against considering Aeromonas as an enteropathogen have been raised. There are some case-control studies that have isolated the micro-organism in symptomatic and asymptomatic patients with an equal incidence, and a single human volunteer challenge study that demonstrated diarrhoea in only two of 57 volunteers [Morgan et al. (1985); incorrectly referenced as Johnson et al. (1985) in the correspondence article of Chu and others]. In contrast, there are many studies in which Aeromonas has been isolated from cases of enteric disease and has disappeared when remission of the symptoms has occurred. Furthermore, additional serological evidence of infection has been provided in other cases, which supports its true enteropathogenicity (see review by Janda \& Abbott, 1998). Although the classic Koch's postulates have not been fulfilled, due to the lack of a suitable animal model for Aeromonas gastrointestinal infection, they have been anecdotally fulfilled by the accidental ingestion of Aeromonas by a healthy laboratory worker, who developed a severe diarrhoea that lasted for 2 days, and from whom the same strain that was ingested was isolated (Carnahan et al., 1991).

Another important aspect to be considered is the risk of Aeromonas bacteraemia when the microbe is colonizing the intestine. A review study, also conducted in Hong Kong, reported on 30 cases of acute suppurative cholangitis produced by Aeromonas, and provided evidence for the ascent of the microbe from the intestine to the biliary tract secondary to the use of instrumentation during exploration (Chan et al., 2000). Haematological spread from the gastrointestinal mucosa is thought to be the most frequent portal of entry of
Aeromonas bacteraemia. The fatality rate in cases of bacteraemia affecting immunocompromised patients may range from 25 to $70 \%$ (Figueras, 2005). Because of this, some authors recommend the surveillance of Aeromonas in faeces of patients with underlying malignancy as a preventive measure for bacteraemia (Janda \& Abbott, 1998).

We hope that this correspondence article will help to increase the awareness of the medical community of the increasingly important role(s) that Aeromonas species may have in human infection. We have known for decades that this group of micro-organisms can be found infecting immunosuppressed patients, but recent publications portray this pathogen as also able to produce severe infections in healthy people, infections that can often be fatal (for a review, see Figueras, 2005). The fact that Aeromonas can act as a true pathogen in cases of cellulitis, necrotizing fasciitis, peritonitis, meningitis and respiratory tract diseases, among others, is never questioned. If this is possible, why is it so difficult to believe that it can produce diarrhoea when the right host and the circumstances concur?

\section{J. Figueras, ${ }^{1}$ A. J. Horneman, ${ }^{2} \dagger$ A. Martinez-Murcia ${ }^{3}$ and J. Guarro ${ }^{1}$}

\section{${ }^{1}$ Unit of Microbiology, Faculty of Medicine, University Rovira and Virgili, 43201 Reus, Spain \\ ${ }^{2}$ Department of Medical and Research Technology, University of Maryland School of Medicine, Baltimore, MD, USA \\ ${ }^{3}$ Molecular Diagnostics Centre (MDC), University Miguel Hernández, Orihuela, Spain}

†Formerly A. J. Martin-Carnahan.

Correspondence: M. J. Figueras (mariajose.figueras@urv.cat)

Albert, M. J., Amarizzaman, M., Tailer, K. A., Chopra, A. K., Kuhn, I., Rahman, M., Faruque, A. S., Islam, M. S., Sack, R. B. \& Molby, R. (2000). Prevalence of enterotoxin genes in Aeromonas spp. isolated from children with diarrhea, healthy controls, and the environment. J Clin Microbiol 38, 3785-3790.

Carnahan, A. M., Chakraborty, T., Fanning, G. R., Verma, D., Ali, A., Janda, J. M. \& Joseph, S. W.
(1991). Aeromonas trota sp. nov., an ampicillinsusceptible species isolated from clinical specimens. J Clin Microbiol 29, 1206-1210.

Chacón, M. R., Figueras, M. J., CastroEscarpulli, G., Soler, L. \& Guarro, J. (2003). Distribution of virulence genes in clinical and environmental isolates of Aeromonas spp. Antonie Van Leeuwenhoek 84, 269-278.

Chan, S. S. \& Ng, K. C. (2004). Aeromonas spp. and infectious diarrhea, Hong Kong. Emerg Infect Dis 10, 1506-1507.

Chan, F. K., Ching, J. Y., Ling, T. K., Chung, S. C. \& Sung, J. J. (2000). Aeromonas infection in acute suppurative cholangitis: review of 30 cases. J Infect 40, 69-73.

Chan, S. S., Ng, K. C., Lyon, D. J., Cheung, W. L., Cheng, A. F. \& Rainer, T. H. (2003). Acute bacterial gastroenteritis: a study of adult patients with positive stool cultures treated in the emergency department. Emerg Med J 20, 335-338.

Chopra, A. K. \& Houston, C. W. (1999).

Enterotoxins in Aeromonas-associated gastroenteritis. Microbes Infect $\mathbf{1}$, 1129-1137.

Chu, Y. W., Wong, C. H., Tsang, G. K., Kwok, M. S., Wong, R. K., Lo, J. Y. \& Kam, K. M. (2006). Lack of association between presentation of diarrhoeal symptoms and faecal isolation of Aeromonas spp. amongst outpatients in Hong Kong. J Med Microbiol 55, 349-351.

Figueras, M. J. (2005). Clinical relevance of Aeromonas. Rev Med Microbiol 16, 145-153.

Galindo, C. L., Sha, J., Fadl, A. A., Pillai, L. \& Chopra, A. K. (2006). Host immune responses to Aeromonas virulence factors. Curr Immunol Rev 2, 13-26.

Hofer, E., Resi, C. M., Theophilo, G. N., Cavalcanti, V. O., Lima, N. V. \& Henriques, M de F. (2006). Aeromonas associated with an acute diarrhea outbreak in Sao Bento do Una, Pernambuco. Rev Soc Bras Med Trop 39, 217-220.

Janda, J. M. \& Abbott, S. L. (1998). Evolving concepts regarding the genus Aeromonas: an expanding panorama of species, disease presentations and unanswered questions. Clin Infect Dis 27, 332-344.

Martin-Carnahan, A. \& Joseph, S. W. (2005). Aeromonas. In Bergey's Manual of Systematic Bacteriology, 2nd edn, vol. 2, pp. 556-578. Edited by D. J. Brenner, N. R. Krieg, J. T. Staley \& G. M. Garrity. New York: Springer.

Morgan, D. R., Johnson, P. C., DuPont, H. L., Satterwhite, T. K. \& Wood, L. V. (1985). Lack of correlation between known virulence properties of Aeromonas hydrophila and enteropathogenicity for humans. Infect Immun 50, 62-65.

Seshadri, R., Joseph, S. W., Chopra, A. K., Sha, J., Shaw, J., Graf, J., Haft, D., Wu, M., Ren, Q. \& other authors (2006). Genome 
sequence of Aeromonas hydrophila ATCC 7966T: jack of all trades. J Bacteriol 188, 8272-8282.

Subashkumar, R., Thayumanavan, T.,

Vivekanandhan, G. \&

Lakshmanaperumalsamy, P. (2006).

Occurrence of Aeromonas hydrophila in acute gastroenteritis among children. Indian J Med Res 123, 61-66.
Teunis, P. F., Nagelkerke, N. J. \& Haas, C. N. (1999). Dose response models for infectious gastroenteritis. Risk Anal 19, 1251-1260.

Vila, J., Ruiz, J., Gallardo, F., Vargas, M., Soler, L., Figueras, M. J. \& Gascon, J. (2003). Aeromonas species and traveler's diarrhea: clinical features and antimicrobial resistance. Emerg Infect Dis 9, 552-555.
Wang, G., Clark, C. G., Liu, C., Pucknell, C., Munro, C. K., Kruk, T. M., Caldeira, R.,

Woodward, D. L. \& Rodgers, F. G. (2003).

Detection and characterization of the hemolysin genes in Aeromonas hydrophila and Aeromonas sobria by multiplex PCR. J Clin Microbiol 41, 1048-1054.

DOI 10.1099/jmm.0.47062-0 